Case Report

\title{
An Intriguing Case of Eosinophilia with FIP1L1/PDGFRA Rearrangement Who Presented as Thrombotic Thrombocytopenic Purpura
}

\author{
Hassan Alshehri (iD, ${ }^{1}$ Mohammad Alnomani, ${ }^{1}$ Mubarak Alghamdi, ${ }^{1}$ Ibrahim Motabi, \\ Imran Tailor ${ }^{(D},{ }^{1}$ Nawal Alshehry, ${ }^{1}$ Mansour Alfayez, ${ }^{1,2}$ Abdul Rehman Z. Zaidi, ${ }^{1}$ \\ Syed Altaf, ${ }^{1}$ Azizah AlSwayyed, ${ }^{3}$ Ammar AlSughayyer, ${ }^{3}$ and Syed Z. A. Zaidi ${ }^{1}$ \\ ${ }^{1}$ Department of Adult Hematology and BMT, King Fahad Medical City, Riyadh, Saudi Arabia \\ ${ }^{2}$ Department of Leukemia, The University of Texas MD Anderson Cancer Center, Houston, TX, USA \\ ${ }^{3}$ Department of Pathology and Clinical Laboratory Medicine, King Fahad Medical City, Riyadh, Saudi Arabia \\ Correspondence should be addressed to Hassan Alshehri; hassan.shehri@gmail.com
}

Received 22 May 2019; Revised 25 August 2019; Accepted 7 September 2019; Published 15 October 2019

Academic Editor: Eduardo Arellano-Rodrigo

Copyright (c) 2019 Hassan Alshehri et al. This is an open access article distributed under the Creative Commons Attribution License, which permits unrestricted use, distribution, and reproduction in any medium, provided the original work is properly cited.

\begin{abstract}
Myeloid neoplasm with eosinophilia and FIP1-like-1-platelet-derived growth factor receptor-alpha (FIP1L1-PDGFRA) rearrangement is a multi-organ disease with diverse clinical presentation. Thrombotic thrombocytopenic purpura (TTP) is characterized by the concomitant occurrence of often severe thrombocytopenia, microangiopathic hemolytic anemia, and a variable degree of ischemic organ damage. To our knowledge, only one case of eosinophilia with FIP1L1-PDGFRA rearrangement presented as a case of thrombotic thrombocytopenic purpura reported in the literature. We herein report a case of a young male patient with hypereosinophilic syndrome and FIP1L1-PDGFRA rearrangement who presented with asthma, transient ischemic attacks (TIA), and confusion. He had an acquired TTP that was successfully treated with plasma exchanges (PLEX), corticosteroids, rituximab, and later with the addition of imatinib mesylate (Gleevec, Novartis). He remains in complete remission on imatinib $100 \mathrm{mg}$ daily for more than 28 months of follow-up.
\end{abstract}

\section{Introduction}

Eosinophilia is commonly observed in diverse nonclonal and clonal disorders. In the majority of cases, it is reactive, associated with atopic conditions, autoimmune disorders, and infections or malignancies, while in other instances, it is a result of an underlying hematologic disorder [1].

Current evaluation of suspected hypereosinophilic syndrome (HES) includes a molecular/cytogenetic investigation with either reverse transcription polymerase chain reaction (RT-PCR) or fluorescent in situ hybridization (FISH) for the FIP1L1-PDGFRA rearrangement $[2,3]$. It is categorized as a specific entity in the 2008 World Health Organization (WHO) classification of myeloid neoplasms (myeloid and lymphoid neoplasms with eosinophilia and abnormalities of PDGFRA, PDGFRB, and FGFR1) [4, 5]. In 2017 revision of WHO classification, myeloid and lymphoid neoplasms associated with $t(8 ; 9)(\mathrm{p} 22 ; \mathrm{p} 24.1)$ and PCM1JAK2 have been recognized as provisional entities to this group of eosinophilic disorders [6]. Tyrosine kinase inhibitors, such as imatinib, are effective in the treatment of FIP1L1-PDGFRA mutated disease. Given the striking sensitivity to imatinib, a dose of $100 \mathrm{mg}$ daily is typically sufficient [7-10].

Thrombotic thrombocytopenic purpura (TTP) is characterized by the concomitant occurrence of often severe thrombocytopenia, microangiopathic hemolytic anemia, and a variable degree of ischemic organ damage, particularly affecting the brain, heart, and kidneys [11]. In acquired TTP, the underlying mechanism is deficiency of metalloprotease 
ADAMTS13 (a disintegrin and metalloproteinase with a thrombospondin type 1 motif, member 13) due to the emergence of its inhibitor.

Simultaneous presentation of HES with FIP1L1PDGFRA rearrangement and TTP is a unique phenomenon. To our knowledge, only one such a case has been reported in the literature [12].

\section{Case Presentation}

A 36-year-old man with past history of 36 pack-years of smoking and bronchial asthma presented to an outside facility on August 11, 2016 with one-hour duration of sudden onset right upper limb weakness that progressed to involve the lower limb along with slurring of speech and altered level of consciousness. CT scan of the brain and carotid ultrasound were reported as normal. His initial complete blood count (CBC) and biochemistry profile showed low hemoglobin and low platelets along with elevated bilirubin. Peripheral blood smear (PBS) showed fragmented red blood cells. TTP was suspected and fresh frozen plasma (FFP) infusion started (due to unavailability of PLEX) along with methylprednisolone intravenously (IV) $500 \mathrm{mg} /$ day. He was then transferred to our facility on August 19, 2016 after 7 days of FFP infusions and methylprednisolone. Upon presentation at our emergency department, he was vitally stable; however, he was intermittently confused with a power of $3 / 5$ in the right upper and lower limbs. His CBC with differential was remarkable for anemia, thrombocytopenia, and leukocytosis with eosinophilia (Table 1). The chest X-ray was normal (Figure 1). Peripheral blood film showed schistocytosis (4\%) with eosinophilia in partly degranulated forms (Figures 2(a)-2)(c). ADAMTS13 level and its inhibitor were sent to the reference laboratory; however, due to delay in processing, the sample was denatured and was not analyzable.

As he presented to our facility, PLEX with cryo-poor plasma 1.5 volumes daily was started along with methylprednisolone IV $500 \mathrm{mg} /$ day for 3 days, followed by prednisolone $1 \mathrm{mg} / \mathrm{kg} /$ day. The patient's right sided weakness and confusion resolved after 2 sessions of PLEX.

As his eosinophilia persisted after giving pulse steroids, we called for a review of his old records. A blood analysis report of July 2012 had shown normal WBC $\left(5.4 \times 10^{9}\right)$ with normal absolute eosinophil count $\left(0.378 \times 10^{9}\right)$. Workup for eosinophilia including stool examination for ova and parasite, schistosoma serology, ANA, and ANCA was reported negative. Fluorescence in situ hybridization (FISH) screening on peripheral blood for FIP1L1-PDGFRA (4q12) rearrangement was positive in $20.5 \%$ of the 200 scored nuclei. T-cell receptor gene rearrangements study was negative.

Bone marrow aspirate and trephine core biopsies were performed. Bone marrow aspirate showed good spicules, and differential count revealed $2 \%$ blasts and 30\% Eosinophils. Megakaryocytes were adequate and morphologically unremarkable. Erythroid and myeloid precursors showed progressive and orderly maturation
(Figures 2(d) and 2(e)). Trephine core biopsy sections showed cellularity of $90 \%$ with trilineage hematopoiesis. Megakaryocytes and erythroid precursors were adequate and morphologically unremarkable. Myeloid precursors were adequate with prominently increased eosinophilic lineage cells (Figure 2(f)). Flow cytometry of the bone marrow (after steroid use) showed a $\mathrm{T}$-cell population (about $78 \%$ of the lymphocytes analyzed) with no aberrant loss or aberrant expression of T-cell markers. Normal male karyotype $(46, \mathrm{XY})$ without chromosomal aberrations was noted on the bone marrow specimen. FISH for $t$ $(9 ; 22)$ (q34; q11) and RT-qPCR for BCR : ABL1 p210 was negative. FISH study done on the bone marrow aspirate for FIP1L1/PDGFRA (4q12) rearrangement detected abnormal signals in $26 \%$ of the 200 scored nuclei (Figure 3).

After 18 sessions of PLEX, his LDH and platelet normalized. At this point, a trial to weaning off PLEX resulted in re-emergence of schistocytes with elevation in $\mathrm{LDH}$ accompanied by a drop-in platelet count, which did necessitate resuming PLEX. Rituximab $375 \mathrm{mg} / \mathrm{m}^{2}$ weekly was started and was given for four doses (August 28, 2016, to September $18,2016)$. Imatinib $100 \mathrm{mg}$ daily was started on September 26, 2016 for steroid-refractory, FIPL1-PDGFRA rearrangement positive eosinophilia. Rapid resolution of his eosinophilia was noted, and absolute eosinophil count dropped from $3.57 \times 10^{9}$ to $0.14 \times 10^{9}$ after 3 days. In total, 30 PLEX sessions were needed to treat the TTP; the last session was 4 days after the initiation of Imatinib (September 30, 2016).

After more than 28 months of follow-up, the patient continues to be in remission, with normal eosinophil count and no TTP recurrence (Figure 4).

\section{Discussion}

Acquired TTP is relatively uncommon but a life-threatening disorder. ADAMTS13 deficiency is most frequently acquired via ADAMTS13 autoantibodies, but rarely, it is inherited via mutations of the ADAMTS13 gene. The first acute episode of TTP usually occurs during adulthood, with a predominant anti-ADAMTS13 autoimmune etiology. Rapid recognition of TTP is crucial to initiate appropriate treatment based on daily therapeutic plasma exchange supplying deficient ADAMTS13 (along with the removal of antibody), with or without steroids. Additional immune modulators targeting ADAMTS13 autoantibodies are mainly based on steroids and the anti-CD20 monoclonal antibody rituximab. In refractory or unresponsive TTP, more intensive therapies including twicedaily plasma exchange; pulses of cyclophosphamide, vincristine, or cyclosporine $A$; or salvage splenectomy may be considered [13]. Multiple reports from Oklahoma $[14,15]$ and other centers, including our facility, were published [13, 16-19]. There are many acquired associations of TTP; however, the co-occurrence of TTP with HES has rarely been reported, and it is even more unique when TTP-related symptoms are the primary presentation [12]. Chaudhary et al. reported unique association of 
TABLE 1: Laboratory characteristics at baseline, after recovery from TTP post-PLEX and post-imatinib therapy.

\begin{tabular}{|c|c|c|c|c|}
\hline Lab parameter & Baseline & $\begin{array}{l}\text { After PLEX (pre- } \\
\text { imatinib) }\end{array}$ & $\begin{array}{l}\text { Post-imatinib } \\
3 \text { months }\end{array}$ & Reference range \\
\hline WBC & 13.62 & 5.78 & 10.57 & $3.90-11.00 \times 10^{9} / \mathrm{L}$ \\
\hline Abs. neutrophils & 7.54 & 3.7 & 4.81 & $1.35-7.50 \times 10^{9} / \mathrm{L}$ \\
\hline Abs. lymphocytes & 2.32 & 1.72 & 5.01 & $1.50-4.30 \times 10^{9} / \mathrm{L}$ \\
\hline Abs. monocytes & 0.65 & 0.35 & 0.58 & $0.25-1.00 \times 10^{9} / \mathrm{L}$ \\
\hline Abs. eosinophils & 3.06 & 1.01 & 0.02 & $0.03-1.00 \times 10^{9} / \mathrm{L}$ \\
\hline Abs. basophils & 0.05 & 0.00 & 0.15 & $0.25-1.00 \times 10^{9} / \mathrm{L}$ \\
\hline Hemoglobin & 7.5 & 11 & 15.1 & $13.50-18.00 \mathrm{~g} / \mathrm{dl}$ \\
\hline Platelet & 30 & 319 & 416 & $155.00-435.00 \times 10^{9} / \mathrm{L}$ \\
\hline Coombs test & Negative & Negative & Negative & \\
\hline \multicolumn{5}{|l|}{ Coagulation profile } \\
\hline aPTT & 34.6 & 31.2 & 35.2 & $28.0-41.0 \mathrm{sec}$ \\
\hline PT & 16.5 & 14.6 & 13.6 & $11.5-15.0 \mathrm{sec}$ \\
\hline INR & 1.3 & 1.1 & 1.0 & $0.9-1.2$ \\
\hline Fibrinogen & 2.6 & 2.2 & 4.8 & $1.4-4.4 \mathrm{~g} / \mathrm{L}$ \\
\hline D-dimer & 2.4 & 0.5 & 0.3 & $0.0-0.5 \mu \mathrm{g} / \mathrm{mL}$ \\
\hline Creatinine & 110 & 83 & 81 & $62-106 \mu \mathrm{mol} / \mathrm{L}$ \\
\hline Urea & 11.6 & 7.2 & 4.4 & $2.5-6.4 \mathrm{mmol} / \mathrm{L}$ \\
\hline Total bilirubin & 94.9 & 5.2 & 10.9 & $0.0-21.0 \mu \mathrm{mol} / \mathrm{L}$ \\
\hline Direct bilirubin & 28.1 & 1.9 & 3.2 & $0.0-3.0 \mu \mathrm{mol} / \mathrm{L}$ \\
\hline LDH & 982 & 230 & 231 & $135-225 \mathrm{U} / 1$ \\
\hline ALT & 25 & 30 & 35 & $0-41 \mathrm{U} / 1$ \\
\hline Alkaline phosphatase & 90 & 86 & 101 & $40-129 \mathrm{U} / 1$ \\
\hline Albumin & 41 & 34 & 39 & $35-50 \mathrm{~g} / \mathrm{L}$ \\
\hline Haptoglobin & $<0.07$ & & & $0.3-1.8 \mathrm{~g} / \mathrm{L}$ \\
\hline $\begin{array}{l}\text { FISH analysis for FIP1L1/PDGFRA } \\
\text { (4q12) rearrangement ( } \% \text { of scored } \\
\text { nuclei) }\end{array}$ & $\begin{array}{l}26 \% \text { in bone marrow and } 20.5 \% \text { in } \\
\text { peripheral blood }\end{array}$ & Not done & Negative & Negative \\
\hline
\end{tabular}

ADAMTS13 level and inhibitor sample were rejected by the reference lab.

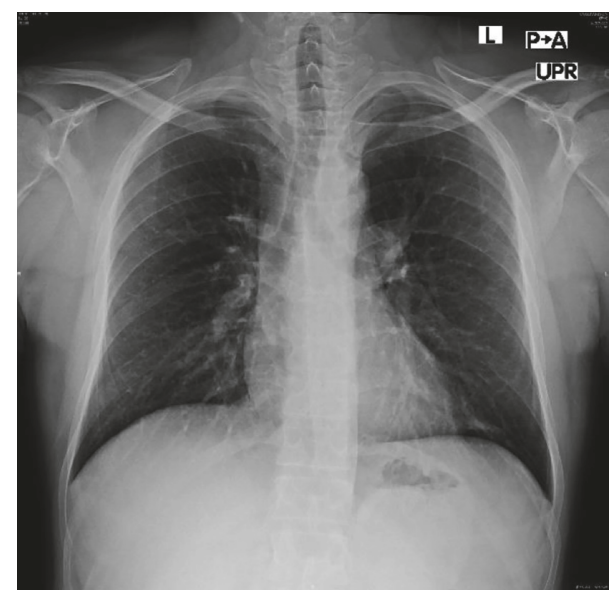

Figure 1: Baseline chest X-ray.

myeloid neoplasm with eosinophilia and abnormalities of PDGFRA with TTP [12]. Their patient was a female in whom TTP presented as one of the earlier manifestations of myeloproliferative HES with rearrangement of PDGFRA. Their patient was found to have a normal ADAMTS13 level which is not commonly seen with TTP [12]. Earlier than this report, two cases with FIP1L1PDGFRA-negative hypereosinophilic syndrome and subsequent TTP have been reported [20, 21]. Ohguchi et al. reported a case of HES who subsequently developed
TTP due to development of ADAMTS13 inhibitor and was successfully treated with corticosteroids and TPE [20]. Al Aly et al. reported a case who developed idiopathic HES [21]. She was treated with imatinib mesylate and subsequently developed TTP with renal failure. A kidney biopsy was performed and was diagnostic of thrombotic microangiopathy. The patient was treated with TPE and hemodialysis. Her eosinophilia resolved, but she remained dialysis-dependent. Our patient was unique as he had refractory TTP and HES. After 18 days of daily PLEX, an attempt of stopping of PLEX was made. Recurrence with increasing LDH and falling platelet count after 6 days was noted. PLEX was resumed on day 7 along with the addition of rituximab for refractory TTP. Meanwhile, we found evidence of FIP1L1-PDGFRA gene rearrangement by FISH analysis. Hence, we added imatinib on September 26, 2016 at a low dose of $100 \mathrm{mg} /$ day. Absolute eosinophil count normalized within 3 days. This rapid response in eosinophil count can be related to concomitant use of steroids. The patient tolerated imatinib well without any adverse events. PLEX was successfully discontinued after a total of 30 PLEX. The patient was maintained on imatinib $100 \mathrm{mg}$ daily and remains in remission for both TTP and HES after more than 28 months of follow-up.

Hypereosinophilic syndromes (HES) constitute a rare and heterogeneous group of disorders, defined as persistent and marked blood eosinophilia $\left(>1.5 \times 10^{9} / \mathrm{L}\right.$ for more than 

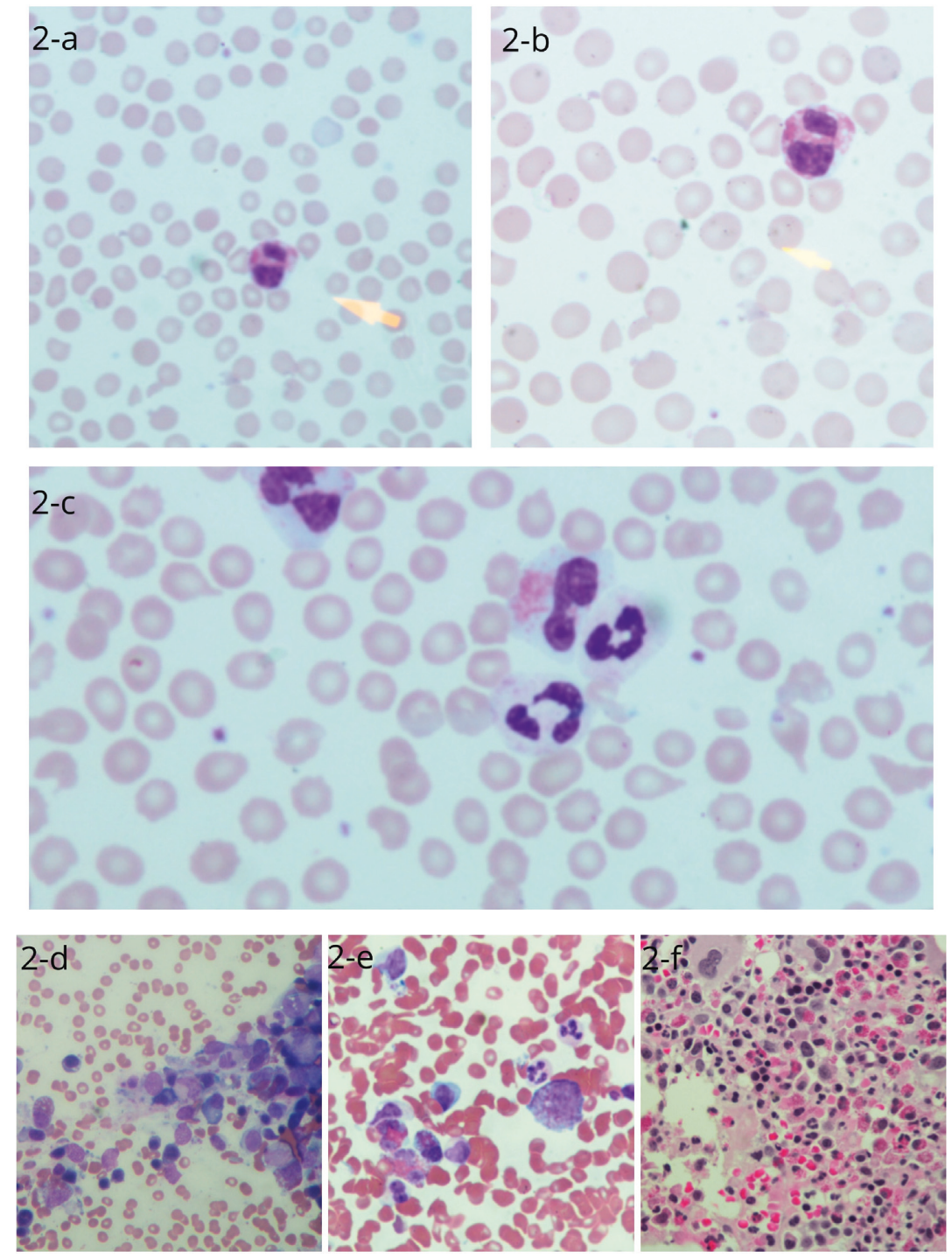

Figure 2: (a, b) Peripheral blood smear revealing schistocytes and eosinophil (May-Grüunwald stain). (c) Partially degranulated eosinophils and schistocytes (May-Grünwald stain). (d, e) Bone marrow aspirate (May-Grünwald stain, 400x magnification). (f) Bone marrow trephine biopsy (hematoxylin-eosin stain, 200x magnification).

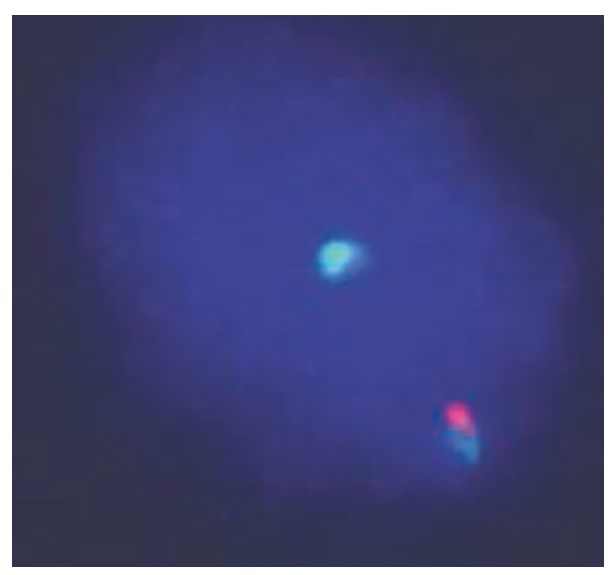

FIGURE 3: Fluorescence in situ hybridization (FISH) analysis for FIP1L1/CHIC/PDGFRA (using Vysis/Abbott FISH probes) revealed FIP1L1/PDGFRA (4q12) rearrangement in $20.5 \%$ of the scored nuclei. ISCN: nuc ish (FIP1L1x2, CHICx1, PDGFRAx2) (FIP1L1 con PDGFRA) (41/200). 


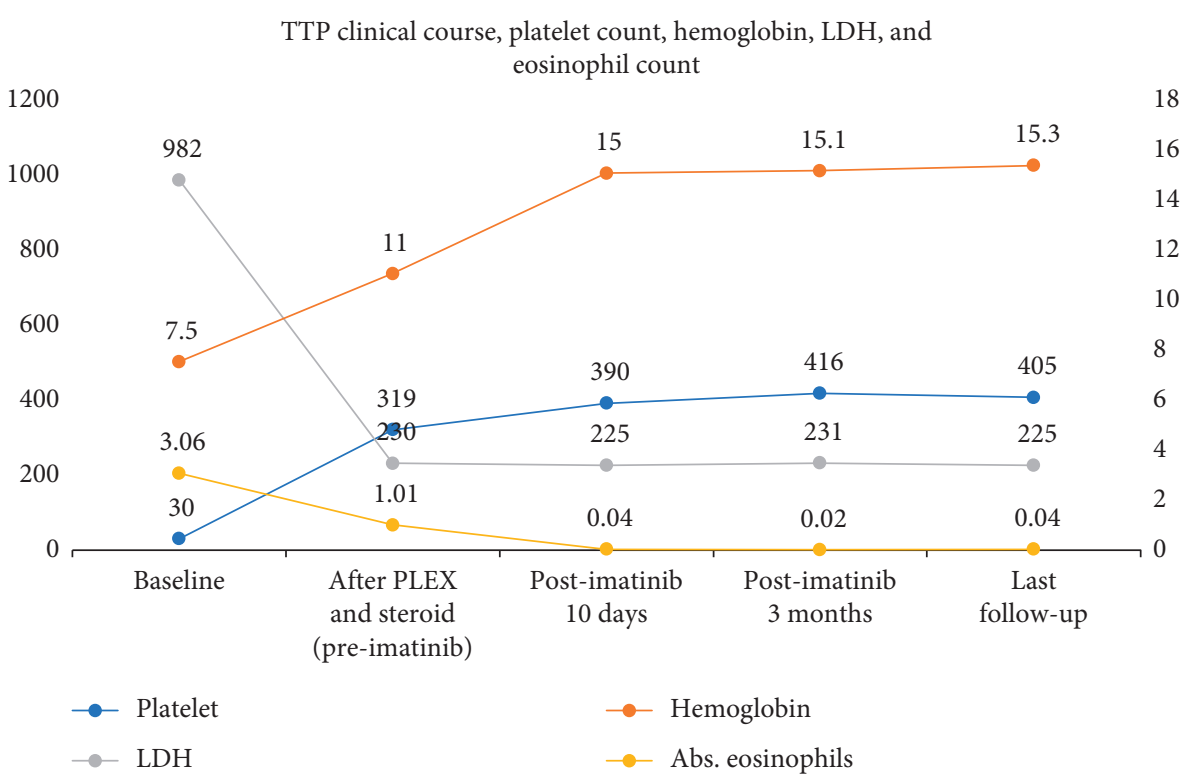

FIGURE 4: Clinical course, platelet count, hemoglobin, LDH, and eosinophil count of the patient. LDH, lactate dehydrogenase; PLEX, plasma exchange.

six consecutive months) associated with evidence of eosinophilinduced organ damage, where other causes of hypereosinophilia such as allergic, parasitic, and malignant disorders have been excluded [22]. Under normal physiologic conditions, eosinophil production is tightly controlled by the cytokine network. The normal eosinophil count in peripheral blood ranges between 0.05 and $0.5 \times 10^{9} / \mathrm{L}$ [23]. It can be reactive (secondary) or an integral phenotype of an underlying hematological neoplasm (primary). The diagnosis of clonal eosinophilia requires the demonstration of either a cytogenetic/ molecular marker of clonality or bone marrow histological features that are not consistent with an otherwise classified myeloid malignancy [3]. FIP1L1 fusions with PDGFRA, PDGFRB, and FGFR1 were identified in 2002-2003 as recurrent rearrangements, with the FIP1L1-PDGFRA fusion being the most common fusion. Karyotype, although less sensitive, can show corresponding gene rearrangement (4q12 (PDGFRA), 5q31-33 (PDGFRB), or 8p11-12 (FGFR1) [24, 25].

Myeloproliferative neoplasms with FIP1L1-PDGFRA highlight the relevance of tyrosine kinases to both normal cellular physiology and disease states. PDGFRA is a member of type III-receptor tyrosine kinases. FIP1L1/PDGFRA protein fusion leads to deregulated PDGFRA kinase activity and abrogates normal growth factor-dependent stimulation of the receptor [26]. Imatinib at a dose of $100 \mathrm{mg}$ is very effective in this disorder.

The plausible mechanism of the simultaneous presentation of TTP and HES is not clear to us. We ponder if neoantigen form FIP1L1-PDGFRA rearmament stimulated an immune response that cross-reacts with ADAMTS13 resulting in its subsequent depletion, or if this is related to eosinophilia/eosinophilic granules causing an independent platelets aggregation or devouring of ADAMTS13. However, the authors could not demonstrate the deficiency of ADAMTS13 or the presence of autoantibodies against ADAMTS13 due to logistic issues discussed. The eosinophilia in our case was clonal in nature; however, in lymphocyte-variant hypereosinophilia or with Th2/cytokines-mediated eosinophilia, TTP, in theory, can be a manifestation of this autoimmune dysregulation.

\section{Conclusion}

To our knowledge, this is the second case report on the simultaneous presentation of TTP with eosinophilia and FIP1L1-PDGFRA rearrangement. This presentation raises the possibility of a mechanistic association between the two disorders. Low dose imatinib resulted in an excellent and sustained response.

\section{Abbreviations}

Abs: $\quad$ Absolute

ADAMTS13: A disintegrin and metalloproteinase with a thrombospondin type 1 motif, member 13

FISH: $\quad$ Fluorescence in situ hybridization

FIP1L1/ FIP1-like-1-platelet-derived growth factor

PDGFRA: receptor-alpha

LDH: $\quad$ Lactate dehydrogenase

PLEX: $\quad$ Plasma exchange

RDW: $\quad$ Red cell distribution width

MCV: $\quad$ Mean corpuscular volume

$\mathrm{MCH}: \quad$ Mean corpuscular hemoglobin.

\section{Conflicts of Interest}

The authors declare that they have no conflicts of interest.

\section{References}

[1] A. Reiter, D. Grimwade, and N. C. P. Cross, "Diagnostic and therapeutic management of eosinophilia-associated chronic 
myeloproliferative disorders," Haematologica, vol. 92, no. 9, pp. 1153-1158, 2007.

[2] G. Loules, F. Kalala, N. Giannakoulas, E. Papadakis, P. Matsouka, and M. Speletas, "FIP1L1-PDGFRA molecular analysis in the differential diagnosis of eosinophilia," $B M C$ Hematology, vol. 9, no. 1, p. 1, 2009.

[3] A. Tefferi, M. M. Patnaik, and A. Pardanani, "Eosinophilia: secondary, clonal and idiopathic," British Journal of Haematology, vol. 133, no. 5, pp. 468-492, 2006.

[4] S. H. C. E. Swerdlow, N. L. Harris, E. S. Jaffe et al., WHO Classification of Tumours of Haematopoietic and Lymphoid Tissues, International Agency for Research on Cancer, Lyon, France, 2008.

[5] A. Tefferi, J. Thiele, and J. W. Vardiman, “The 2008 World Health Organization classification system for myeloproliferative neoplasms," Cancer, vol. 115, no. 17, pp. 3842-3847, 2009.

[6] B. J. H. H.-P. Bain, D. A. Arber, A. Tefferi et al., WHO Classification of Tumours of Haematopoietic and Lymphoid Tissues, International Agency for Research on Cancer, Lyon, France, 4th edition, 2017.

[7] J. V. Jovanovic, J. Score, K. Waghorn et al., "Low-dose imatinib mesylate leads to rapid induction of major molecular responses and achievement of complete molecular remission in FIP1L1-PDGFRA positive chronic eosinophilic leukemia," Blood, vol. 109, no. 11, pp. 4635-4640, 2007.

[8] J. Cortes, P. Ault, C. Koller et al., "Efficacy of imatinib mesylate in the treatment of idiopathic hypereosinophilic syndrome," Blood, vol. 101, no. >12, pp. 4714-4716, 2003.

[9] M. Baccarani, D. Cilloni, M. Rondoni et al., "The efficacy of imatinib mesylate in patients with FIP1L1-PDGFR -positive hypereosinophilic syndrome. Results of a multicenter prospective study," Haematologica, vol. 92, no. 9, pp. 1173-1179, 2007.

[10] A. Pardanani, S. R. Brockman, S. F. Paternoster et al., "FIP1L1-PDGFRA fusion: prevalence and clinicopathologic correlates in 89 consecutive patients with moderate to severe eosinophilia," Blood, vol. 104, no. 10, pp. 3038-3045, 2004.

[11] J. A. Kremer Hovinga, P. Coppo, B. Lämmle, J. L. Moake, T. Miyata, and K. Vanhoorelbeke, "Thrombotic thrombocytopenic purpura," Nature Reviews Disease Primers, vol. 3, no. 1, p. 17020, 2017.

[12] L. N. Chaudhary, N. G. Bailey, J. A. Vos, and C. J. Stotler, "Unique association of myeloid neoplasm with eosinophilia and abnormalities of PDGFRA with TTP," The West Virginia Medical Journal, vol. 109, no. 2, pp. 6-9, 2013.

[13] B. S. Joly, P. Coppo, and A. Veyradier, "Thrombotic thrombocytopenic purpura," Blood, vol. 129, no. 21, pp. 2836-2846, 2017.

[14] J. N. George, S. K. Vesely, D. R. Terrell et al., “The Oklahoma thrombotic thrombocytopenic purpura-haemolytic uraemic syndrome registry. A model for clinical research, education and patient care," Hämostaseologie, vol. 33, no. 2, pp. 105-112, 2013.

[15] J. N. George, D. R. Terrell, K. K. Swisher, and S. K. Vesely, "Lessons learned from the Oklahoma thrombotic thrombocytopenic purpura-hemolytic uremic syndrome registry," Journal of Clinical Apheresis, vol. 23, no. 4, pp. 129-137, 2008.

[16] E. E. Page, J. A. Kremer Hovinga, D. R. Terrell, S. K. Vesely, and J. N. George, "Thrombotic thrombocytopenic purpura: diagnostic criteria, clinical features, and long-term outcomes from 1995 through 2015," Blood Advances, vol. 1, no. 10, pp. 590-600, 2017.
[17] J. N. George, "The remarkable diversity of thrombotic thrombocytopenic purpura: a perspective," Blood Advances, vol. 2, no. 12, pp. 1510-1516, 2018.

[18] J. N. George, "TTP: long-term outcomes following recovery," Hematology, Hematology American Society of Hematology Education Program, vol. 2018, no. 1, pp. 548-552, 2018.

[19] S. Iqbal, S. Z. Zaidi, I. H. Motabi, N. F. Alshehry, M. S. AlGhamdi, and I. K. Tailor, "Thrombotic thrombocytopenic purpura-analysis of clinical features, laboratory characteristics and therapeutic outcomeof 24 patients treated at a Tertiary Care Center in Saudi Arabia," Pakistan Journal of Medical Sciences, vol. 32, no. 6, pp. 1494-1499, 2016.

[20] H. Ohguchi, T. Sugawara, and H. Harigae, "Thrombotic thrombocytopenic purpura complicated with hypereosinophilic syndrome," Internal Medicine, vol. 48, no. 18, pp. 1687-1690, 2009.

[21] Z. Al Aly, J. M. Philoctête Ashley, M. E. Gellens, and E. A. González, "Thrombotic thrombocytopenic purpura in a patient treated with imatinib mesylate: true association or mere coincidence?," American Journal of Kidney Diseases, vol. 45, no. 4, pp. 762-768, 2005.

[22] F. E. Roufosse, M. Goldman, and E. Cogan, "Hypereosinophilic syndromes," Orphanet Journal of Rare Diseases, vol. 2, no. 1, p. 37, 2007.

[23] P. Valent, A. D. Klion, H.-P. Horny et al., "Contemporary consensus proposal on criteria and classification of eosinophilic disorders and related syndromes," Journal of Allergy and Clinical Immunology, vol. 130, no. 3, pp. 607-612, 2012.

[24] J. Gotlib and J. Cools, "Five years since the discovery of FIP1L1-PDGFRA: what we have learned about the fusion and other molecularly defined eosinophilias," Leukemia, vol. 22, no. 11, pp. 1999-2010, 2008.

[25] B. J. Bain, "Myeloid and lymphoid neoplasms with eosinophilia and abnormalities of PDGFRA, PDGFRB or FGFR1," Haematologica, vol. 95, no. 5, pp. 696-698, 2010.

[26] S. Coutré and J. Gotlib, "Targeted treatment of hypereosinophilic syndromes and chronic eosinophilic leukemias with imatinib mesylate," Seminars in Cancer Biology, vol. 14, no. 4, pp. 307-315, 2004. 


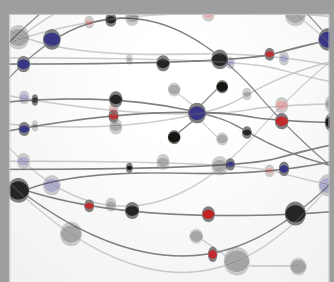

The Scientific World Journal
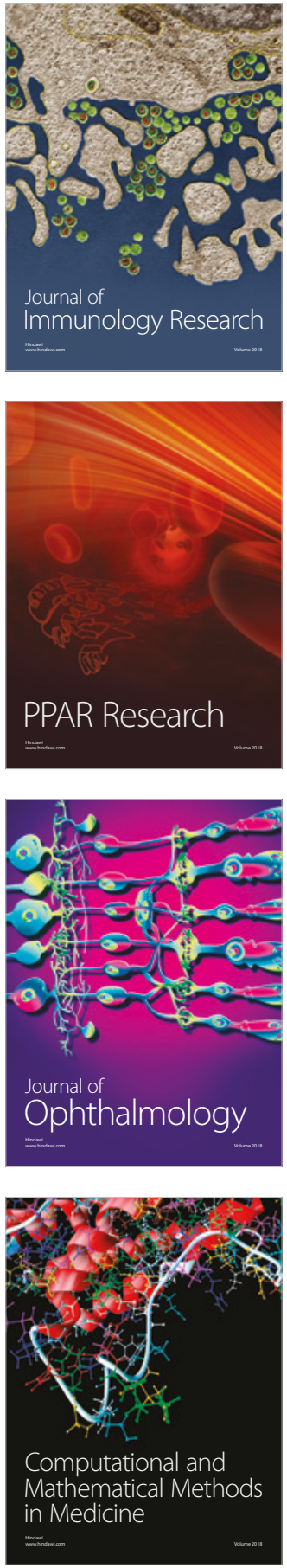

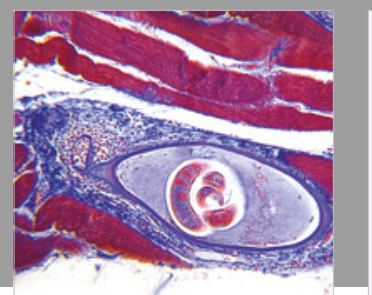

Gastroenterology Research and Practice

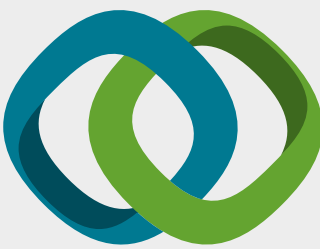

\section{Hindawi}

Submit your manuscripts at

www.hindawi.com
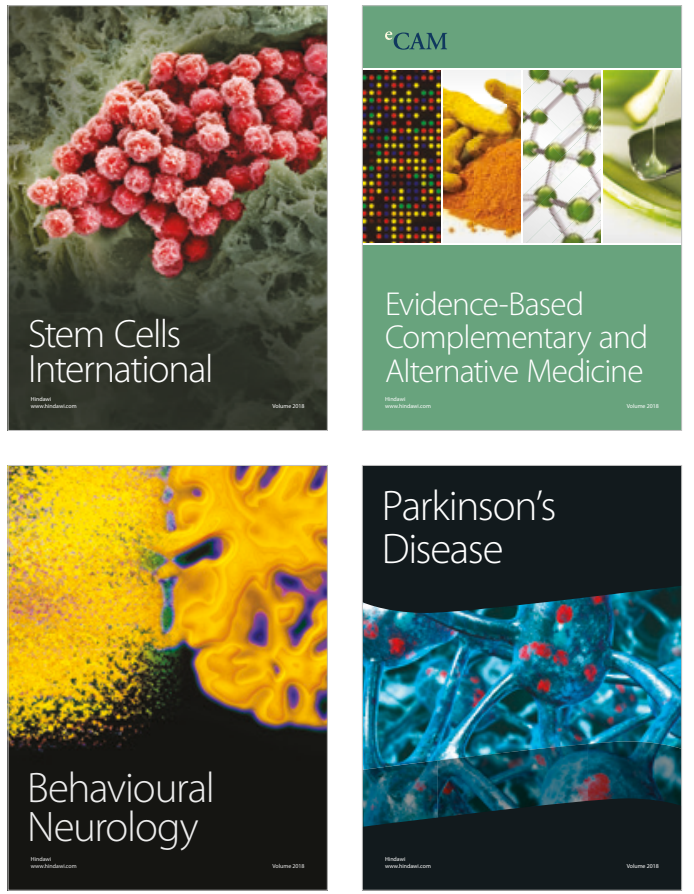



ournal of

Diabetes Research

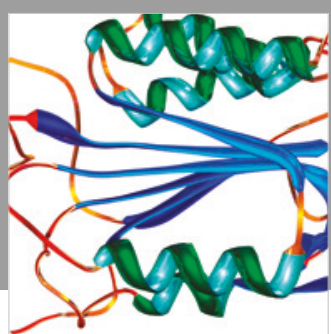

Disease Markers
